\title{
Inhibition of rotavirus replication by a non-neutralizing, rotavirus VP6-specific IgA mAb
}

\author{
Ningguo Feng, ${ }^{1}$ Jeffrey A. Lawton, ${ }^{2}$ Joana Gilbert, ${ }^{1}$ Nelly Kuklin, ${ }^{1}$ Phuoc Vo, ${ }^{1}$ \\ B.V. Venkataram Prasad, ${ }^{2}$ and Harry B. Greenberg ${ }^{1,3}$ \\ ${ }^{1}$ Department of Gastroenterology, Microbiology, and Immunology, Stanford University School of Medicine, \\ Stanford, California, USA \\ ${ }^{2}$ Verna and Maars McLean Department of Biochemistry and Molecular Biology, Baylor College of Medicine, \\ Houston, Texas, USA \\ ${ }^{3}$ Veterans Administration Palo Alto Health Care System, Palo Alto, California, USA \\ Address correspondence to: Harry B. Greenberg, Department of Gastroenterology, Stanford University, 3801 Miranda Avenue, \\ 154-C, Palo Alto, California 94304, USA. Phone: (650) 919-3711; Fax: (650) 852-3259; E-mail: hgreenberg@aviron.com. \\ Jeffrey A. Lawton's present address is: Department of Chemistry and Biochemistry, University of California at San Diego, \\ San Diego, California, USA. \\ Ningguo Feng and Jeffrey A. Lawton contributed equally to this work.
}

Received for publication October 11, 2001, and accepted in revised form March 25, 2002.

Rotaviruses are the leading cause of severe diarrheal disease in young children. Intestinal mucosal
IgA responses play a critical role in protective immunity against rotavirus reinfection. Rotaviruses
consist of three concentric capsid layers surrounding a genome of 11 segments of double-stranded
RNA. The outer layer proteins, VP4 and VP7, which are responsible for viral attachment and entry,
are targets for protective neutralizing antibodies. However, IgA mAb's directed against the interme-
diate capsid protein VP6, which do not neutralize the virus, have also been shown to protect mice
from rotavirus infection and clear chronic infection in SCID mice. We investigated whether the anti-
VP6 IgA (7D9) mAb could inhibit rotavirus replication inside epithelial cells and found that 7D9
acted at an early stage of infection to neutralize rotavirus following antibody lipofection. Using elec-
tron cryomicroscopy, we determined the three-dimensional structure of the virus-antibody complex.
The attachment of 7D9 IgA to VP6 introduces a conformational change in the VP6 trimer, rendering
the particle transcriptionally incompetent and preventing the elongation of initiated transcripts.
Based on these observations, we suggest that anti-VP6 IgA antibodies confers protection in vivo by
inhibiting viral transcription at the start of the intracellular phase of the viral replication cycle. J. Clin. Invest. 109:1203-1213 (2002). DOI:10.1172/JCI200214397.

\section{Introduction}

Rotavirus is the most important cause of severe dehydrating diarrhea in infants and young children worldwide. Regardless of the social and economic status, nearly all children will be infected with rotavirus before 3 years of age. Over 500,000 children, primarily from developing countries, die every year from rotavirus infection, and many more have severe diarrhea that requires hospitalization (1). Given the severity and scope of rotavirus infection, there is an urgent need for a safe and effective vaccine.

Rotaviruses have a complex architecture. The viral capsid is composed of three concentric protein layers surrounding a genome of 11 segments of double-stranded RNA. The outer capsid layer consists of VP4 and VP7, the intermediate layer is composed of VP6, and the viral core is made up of a shell protein VP2 as well as enzymes VP1 and VP3. The viral core contains all of the enzymatic activities needed for the synthesis of full-length, capped mRNA transcripts of the 11-genome segments (2).

The viral outer capsid proteins, VP4 and VP7, are responsible for virus attachment and entry into susceptible cells. These proteins also determine virus serotype and mediate neutralization (3). Antibodies against either VP4 or VP7 can inhibit rotavirus growth in a serotype-specific and heterotypic manner both in vitro and in vivo (4-7). Therefore VP4 and VP7 have long been considered the targets for protective immunity in vaccine development. However, several human and animal studies suggest that VP4 and VP7 may not be the only targets for protective immune responses. Protection rendered by either natural infection or immunization is not strictly serotype-specific (8). Levels of neutralizing antibody responses are not always correlated with protection $(9,10)$. In an animal model, mice immunized with simian-human reassortant rotaviruses are protected against a serotype 3 murine virus re-infection (11). Immunization with VP2/ 6 viruslike particles, VP6 DNA, and VP6 peptides provides protection in various animal models (12-16). These results indicate that other viral proteins may also play a role in induction of the protective immune response.

Virus intermediate layer protein VP6 contains several highly conserved group-reactive epitopes. Although it is the most antigenic viral protein, antibodies against VP6 do not inhibit virus replication in conventional in 
vitro neutralization assays and do not protect animals when fed orally (17). However, some anti-VP6 IgA mAb's such as 7D9 have been shown to protect nonimmune mice from infection and clear chronic infection in SCID mice (17). These anti-VP6 antibodies have no neutralizing activity in vitro or in vivo when fed orally; hence it is unlikely that they inhibit virus by blocking viral binding or entry into host cells.

During the rotavirus replication cycle, virions attach to host cells as triple-layered particles (TLPs) and subsequently enter the cytoplasm by either plasma membrane or endosomal membrane penetration (3). As a result of cell entry, the outer layer of VP4/VP7 is lost, and the resulting double-layered particles (DLPs) become transcriptionally active, releasing mRNA transcripts through a system of channels that penetrate the VP6 and inner VP2 capsid layers at each of the icosahedral vertices (18).

Because mRNA production only occurs within intact subviral particles, it is not surprising that the capsid proteins themselves play important roles in facilitating transcription. The loss of the outermost capsid layer at cell entry and the integrity of the intermediate VP6 capsid layer are both required for the production of mRNA transcripts $(19,20)$. Mature TLPs are able to initiate transcription but are unable to elongate nascent transcripts beyond 6-7 bases in length (21). Although the reason for this is unclear, a growing body of evidence suggests that the attachment of ligands, such as the VP7 capsid protein or certain mAb's, to specific locations on the surface of VP6 causes a subtle architectural rearrangement within the VP6 capsid layer, altering the conformation and reducing the enzymatic activity of the viral RNA polymerase in the core $(21,22)$. These studies suggest that the interaction of anti-VP6 IgA antibodies with VP6 could potentially affect the efficient production of viral mRNA transcripts.

Secretory $\operatorname{IgA}(\mathrm{s} \operatorname{IgA})$ is the predominant antibody isotype present on mucosal surfaces such as the gastrointestinal, respiratory, and genitourinary tracts. Mucosal epithelial cells have IgA-specific polymeric Ig receptors on their basal membrane surfaces, which bind to IgA, transport the antibody to the apical side of cells, and release it into the lumen (23). This process, which is called sIgA transcytosis, ensures a high concentration of sIgA on the mucosal surface. Since anti-VP6 IgA antibodies do not protect when fed orally or neutralize in traditional neutralization assays, we hypothesized that the protective IgA antibodies might "neutralize" rotavirus by interacting with viral DLPs inside enterocytes during transcytosis and by inhibiting the viral replication at the stage of RNA transcription.

In this study we used an in vitro lipofection-mediated intracellular neutralization assay to further investigate possible mechanisms of the 7D9 VP6 IgA mAb protection. We used an in vitro rotavirus mRNA transcription assay to demonstrate that a protective VP6specific IgA mAb 7D9 neutralized different strains of rotaviruses when the antibody and virus DLPs were cotransfected inside cells, and that the antibody also efficiently inhibited rotavirus transcription. This intracellular neutralization activity occurred at an early stage of rotavirus infection.

To further understand the mechanism by which antiVP6 IgA antibodies may be able to prevent viral replication in vivo, we determined the three-dimensional structure of the rotavirus DLP complexed with bivalent 7D9 IgA antibodies to a resolution of about $21 \AA ̊$ using electron cryomicroscopy and image reconstruction. We observed that the attachment of 7D9 to the VP6 surface introduced significant conformational changes within the body of the VP6 capsid layer. Though more substantial, these architectural changes were similar overall to those observed in the VP6 capsid when VP7 is attached. Likewise, biochemical characterization of the DLP:IgA complexes showed that antibody attachment mimicked the inhibitory effect of the capsid protein VP7 in the mature particle, causing a nearly complete inhibition of transcript elongation beyond 6-7 bases. These results provide further insight into the manner by which anti-VP6 IgA antibodies may confer protection against rotavirus infection in vivo.

\section{Methods}

Cells and viruses. Tissue culture-adapted murine rotavirus EC,VP7 serotype 3 and VP4 genotype 16 $(3,[16])$, semian rotavirus RRV (3,[3]), SA11-4F (3,[1]), human rotavirus Wa $(1,[8])$, and bovine rotavirus $\operatorname{NCDV}(5,[1])$ were propagated in the MA104 green monkey kidney cell line as previous described (11). MA104 cells were maintained in media M199 (BioWhittaker Inc., Walkersville, Maryland, USA) with penicillin/streptomycin (M199, GIBCO BRL; Life Technologies Inc., Grand Island, New York, USA) and supplemented with 7\% FCS. Virus culture yields were freeze-thawed twice, and rotaviruses were either concentrated by centrifugation at $140,000 \mathrm{~g}$ for 2 hours through a $40 \%$ sucrose cushion for neutralization assays (see below) or purified on a cesium chloride density gradient for RNA transcription assays (24). To make rotavirus DLPs, purified or concentrated virus was treated with $5 \mathrm{mM}$ EDTA and incubated at $37^{\circ} \mathrm{C}$ for 20 minutes and then dialyzed against PBS without $\mathrm{Ca}^{++}$and $\mathrm{Mg}^{++}$. The resulting DLP preparation was not infectious (25) when directly inoculated on MA104 cells at a concentration greater than or equal to tenfold more than that used in the lipofection-mediated neutralization assay (see below). Propagation and $\mathrm{ID}_{50}$ assays of a wild-type murine rotavirus $\mathrm{ECw}$ were described previously (26).

$m A b$ 's. The mAb's 7D9 and 8D3 (VP6-specific, IgA), 1E11 and SGI-255/60 (VP6-specific, IgG), B4-2 (NSP4specific, IgG), and 159 (VP7-specific, IgG) have been described previously $(4,17,27,28)$. All mAb's used in neutralization assays and RNA transcription assays were purified from ascites using protein L (CLONTECH Laboratories Inc., Palo Alto, California, USA) or protein A (Pierce Chemical Co., Rockford, Illinois, 
USA), dialyzed against PBS and stored in PBS. Antibody concentrations of purified mAb's or ascites were determined by ELISA. Polyethylene microtiter plates were coated with goat anti-mouse IgG, A, and M (Kirkegaard \& Perry Laboratories Inc., Gaithersburg, Maryland, USA) at $4{ }^{\circ} \mathrm{C}$ overnight and then blocked with 5\% dry skim milk (Carnation; Nestle USA Inc., Solon, Ohio, USA) in PBS. After washing with PBS- $0.05 \%$ Tween-20 (PBS-T), serial dilutions of purified $\mathrm{mAb}$ or ascites were added to the plates. IgG or IgA standards (purified mouse IgG [Sigma Chemical Co., St. Louis, Missouri, USA] or mouse IgA myeloma protein [Sigma Chemical Co.]) were also added to each plate. Plates were incubated at $37^{\circ} \mathrm{C}$ for 2 hours. After incubation, plates were washed with PBS-T and peroxidase-conjugated goat anti-mouse IgG ( $\gamma$ chain-specific; Kirkegaard \& Perry Laboratories Inc.) or IgA ( $\alpha$ chain-specific; Kirkegaard \& Perry Laboratories Inc.) antibody was added. After a 1-hour incubation at $37^{\circ} \mathrm{C}$, plates were washed and the color reaction was developed with 2,2'-azino-di-(3-ethylbenzthiazoline-6sulfonate) (ABTS) substrate (Kirkegaard \& Perry Laboratories Inc.). OD was measured on a microtiter plate reader (Bio-Tek Instruments Inc., Winooski, Vermont, USA) at wavelength of $405 \mathrm{~nm}$. Concentrations of the $\mathrm{mAb}$ preparations were calculated based on the standard curves generated from purified mouse IgG or IgA standards and adjusted to $1 \mathrm{mg} / \mathrm{ml}$ for purified $\mathrm{mAb}$ 's and $3 \mathrm{mg} / \mathrm{ml}$ for ascites.

Lipofection-mediated neutralization assay. Classical focus reduction neutralization assay for rotavirus was carried out as described (29). Infection of MA104 cells with rotavirus DLPs using lipofectin-mediated transfection was carried out as described (30). The lipofection-mediated intracellular neutralization assay was based on the rotavirus DLP transfection method. Rotavirus DLPs diluted in M199 (to give approximately 50-100 foci per field in an "unneutralized" control using an inverted microscope) were mixed with lipofectin (GIBCO BRL; Life Technologies Inc.) (15\% vol/vol) and incubated at room temperature for 40 minutes. Selected mAb's (starting concentration $1 \mathrm{mg} / \mathrm{ml}$ ) were diluted fourfold from 1:40 to 1:163,840 in M199 and added at $30 \mu \mathrm{l}$ per well to 96-well plates. An equal volume of lipofectin-treated DLPs was added to the 96-well plates and incubated at $37^{\circ} \mathrm{C}$ for 1 hour. The mixtures were then transferred to MA104 cell monolayers in 96-well plates ( $40 \mu \mathrm{l}$ per well) and incubated at $37^{\circ} \mathrm{C}$ and $5 \% \mathrm{CO}_{2}$ for 4 hours. An additional $40 \mu \mathrm{l}$ of M199 with $20 \%$ FCS was then added to each well, and the incubation was continued for 6 hours. The plates were then washed once and fixed with cold methanol. Fixed plates were washed with HBSS. Rabbit anti-rotavirus hyperimmune serum was added to the plates and incubated for 1 hour at $37^{\circ} \mathrm{C}$. Plates were then washed, and peroxidase-conjugated goat anti-rabbit IgG antibody (Kirkegaard \& Perry Laboratories Inc.) was added for 1 hour at $37^{\circ} \mathrm{C}$. After washing, the color reaction was developed with 3-amino-9-ethylcarbazole substrate, and viral antigen-positive foci were counted in each field as above. The neutralization titer of an antibody was defined as the highest dilution at which there was a $>50 \%$ reduction of viral antigen-positive foci as compared with the no-antibody control.

Quantitation of intracellular antibody. Four hours after transfection of the virus-antibody mixture by lipofectin (see above), MA104 cells were fixed with cold methanol and permeabilized with $1 \%$ Triton $X$ in PBS. Peroxidase-conjugated goat anti-mouse IgG ( $\gamma$ chain-specific) or IgA ( $\alpha$ chain-specific) was added for 1 hour at $37^{\circ} \mathrm{C}$. The plates were then washed five times, and ABTS substrate was added. After 10-15 minutes of color development, the reaction mixture was transferred to a polyethylene ELISA plate and the absorbance measured with a microtiter plate reader. As a control, DLPs and antibodies with identical dilution were added to separate wells in the same plate without lipofectin and were processed and stained in the same fashion. Intracellular antibody titers in transfected wells were expressed as the highest dilution that had a specific OD greater than 0.1. The specific OD was defined as the OD from antibody-transfected well minus the OD from control wells. The OD readings from nontransfected control wells were not significantly different from those from wells with MA104 cell alone without antibody added.

Administration of $m A b$ 's and wild-type rotavirus challenge in adult mice. Six- to eight-week-old female BALB/c mice were injected intraperitoneally twice per day with $500 \mu \mathrm{l}$ of ascites (total daily dose of $3 \mathrm{mg}$ of antibody) from 2 days before challenge until 7 days after challenge. Two days after the initial inoculation (total 4 doses), mice were orally inoculated with $100 \mu \mathrm{l}$ of $1.33 \%$ sodium bicarbonate followed by $10^{2} \mathrm{ID}_{50}$ of ECw. Fecal samples were collected from 1 day before challenge until 7 days after challenge. Fecal rotavirus antigen shedding was measured by ELISA as previously described (26). The area under the antigen shedding curve was calculated as total viral antigen shedding (TVS) (26). The TVS was used as the measure of shedding for statistical analysis. Percentage reduction of fecal virus shedding as compared with control group for each antibody-treated mouse was calculated as (mean TVS of control group TVS of treated mouse $) \div$ mean TVS of control group.

Inbibition of transcription assay. For the studies of the ability of different mAb's to inhibit rotavirus transcription (Figure 1) we used an in vitro transcription assay (31). Purified EC or RRV DLPs were activated with 5 mM EDTA at $37^{\circ} \mathrm{C}$ for 30 minutes. Equal volumes of EDTA-activated viruses and purified antibodies were mixed and incubated at $37^{\circ} \mathrm{C}$ for 60 minutes. Rotavirus in vitro transcription was initially performed using a Riboprobe system (Promega Corp., Madison, Wisconsin, USA) (including optimized $\times 5$ transcription buffer, $100 \mathrm{mM}$ DTT, recombinant RNasin ribonuclease inhibitor, and rNTP) according to the manufacturer's instructions. Transcription products were labeled with ${ }^{32} \mathrm{P} \mathrm{rCTP}(50 \mu \mathrm{Ci})$, and the reaction was carried at $40^{\circ} \mathrm{C}$ for 1 hour. Labeled viral RNA was precipitated with cold 
trichloroacetic acid and absorbed on filter paper disks as described in the protocol for the Riboprobe system. The filter paper disks were washed extensively with cold trichloroacetic acid and air-dried. The incorporated ${ }^{32} \mathrm{P}$ was measured by a Wallac MicroBeta TriLux liquid scintillation counter (Wallac Inc., Turku, Finland). Data were expressed as cpm.

Electron cryomicroscopy. Complexes of DLP with 7D9 $\operatorname{IgA}$ were formed by mixing DLPs and $\operatorname{IgA}$ in a stoichiometric ratio equivalent to five antigen-binding regions per VP6 molecule in order to ensure complete saturation of all available epitopes. Such a molar excess of IgA should inhibit viral aggregation. Mixtures were incubated for 2 hours at room temperature and then concentrated to about $10^{12}$ particles per milliliter by ultrafiltration using a MicroCon-100 microconcentrator (Millipore Corp., Bedford, Massachusetts, USA). Specimens for microscopy were embedded in vitreous ice on holey carbon films according to standard procedures (32).

Specimens were observed on a JEOL 1200EX transmission electron microscope (JEOL USA Inc., Peabody, Massachusetts, USA) operated at $\times 30,000$ magnification using 100 kilo-electron volts electrons and a beam dose of approximately 5 electrons/ $\AA^{2}$. Images were collected using Kodak SO-163 electron film (Eastman Kodak Co., Rochester, New York, USA) with a 1-second exposure time. Micrographs were developed for 12 minutes using Kodak D-19 developer at $21^{\circ} \mathrm{C}$ and fixed for 10 minutes in Kodak Fixer (Eastman Kodak Co.). For each imaging field, two micrographs were collected, one at about $1 \mu \mathrm{m}$ underfocus and the other at about $2 \mu \mathrm{m}$ underfocus.

Three-dimensional structural analysis. Micrographs suitable for three-dimensional structural analysis were digitized using a Perkin-Elmer 1010M microdensitometer (Perkin-Elmer Inc., Shelton, Connecticut, USA) operated at a raster scanning interval of $5.33 \AA$ A per pixel in the object. Three-dimensional reconstructions were produced using software in the ICOS Toolkit suite (33) according to standard procedures (34-37). Particle centers and orientations were initially estimated from further-from-focus image data, and the parameters were then applied to the closer-to-focus image set for refinement and structural determination. The three-dimensional reconstruction was computed to a resolution of about $21 \AA$ Asing 137 particle images. The effects of the contrast transfer function were corrected as previously described (38), assuming $10 \%$ amplitude contrast and a noise-to-signal ratio of 0.3 . The resolution achieved in the final structure was confirmed by Fourier ring correlation analysis (39). The distribution of image data throughout the asymmetric unit was sufficient for the resolution achieved, as $97 \%$ of the mean inverse eigenvalue spectrum was less than 0.01 .

The three-dimensional structure was viewed using IRIS Explorer 3.5 (Numerical Algorithms Group Ltd., Oxford, United Kingdom) on a Silicon Graphics Indigo $^{2}$ (Silicon Graphics Inc., Mountain View, California,

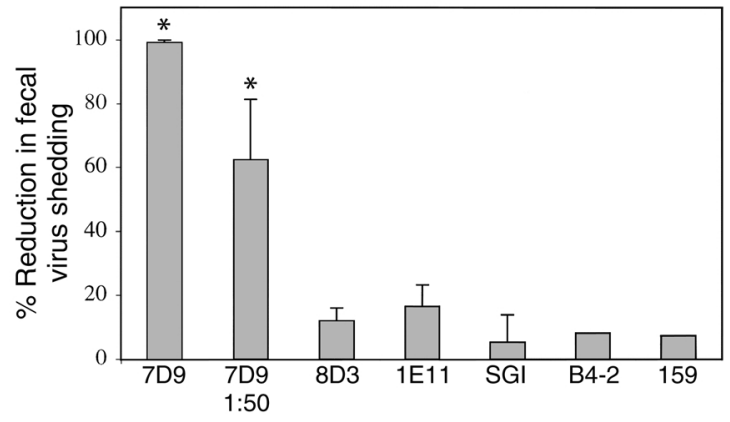

\section{Figure 1}

Protective efficacy following intraperitoneal injection of selected rotavirus-specific mAb's. BALB/c female mice (three mice per group) were intraperitoneally injected with $\mathrm{mAb}$ 's $(3 \mathrm{mg} / \mathrm{d})$ twice a day starting 2 days prior to $10^{2} I D_{50} E C w$ challenge (see Methods). Treatment continued until day 7 after challenge. Fecal virus shedding for each mouse was detected by ELISA, and the area under the 7-day antigen shedding curve was calculated. Figure shows the mean percentage reduction of virus shedding for each group + SEM. *Percentage reduction in these 7D9-inoculated groups was significantly different from that in non-7D9-inoculated groups $(P<0.05)$. Results represent two separate experiments.

USA) workstation. Surface representations were displayed using mass density threshold chosen to account for the expected number of VP6 molecules within the VP6 capsid region. To compare internal regions of mass between native and antibody-bound structures, mass density values were converted to variance and the maps were scaled to have the same minima and maxima. As with the surface representations, the surface contour level was chosen to account for the expected number of VP6 molecules within the VP6 capsid region, and contour intervals were chosen to represent density differences of about $0.37 \sigma$.

Analysis of transcription products. A second transcription assay was used to examine the effect of 7D9 on elongation (see Figure 6). Complexes of DLP with 7D9 IgA were formed by mixing purified DLPs and IgAs in a stoichiometric ratio equivalent to three antigenbinding regions per VP6 molecule. The mixture was incubated at room temperature for 2 hours and then sonicated for 1 minute. Virus:IgA complexes derived from $5 \mu \mathrm{g}$ DLP were suspended in a standard transcription reaction buffer $(30 \mu \mathrm{l})$ containing $100 \mathrm{mM}$ Tris. $\mathrm{HCl}(\mathrm{pH}$ 8), $10 \mathrm{mM} \mathrm{MgOAc}, 0.5 \mathrm{mM} S$-adenosylmethionine, $1 \mathrm{mM}$ ATP, $100 \mu \mathrm{M}$ each of GTP, CTP, and UTP, and $40 \mu \mathrm{Ci}\left[\alpha_{-}{ }^{32} \mathrm{P}\right] \mathrm{GTP}(3000 \mathrm{Ci} / \mathrm{mmol}$; Amersham Pharmacia Biotech, Piscataway, New Jersey, USA) and incubated at $37^{\circ} \mathrm{C}$ for 20 minutes. The reaction mixture was passed through a Micro Bio-Spin P-30 column (Bio-Rad Laboratories Inc., Hercules, California, USA) equilibrated in $10 \mathrm{mM}$ Tris buffer ( $\mathrm{pH} 7$ ) to remove salt and unincorporated radionucleotides. The eluate was lyophilized and resuspended in $1 \times$ TrisBorate-EDTA sample buffer containing $7 \mathrm{M}$ urea and $0.1 \%$ SDS and boiled for 5 minutes to disrupt the particles and denature the nucleic acid. The transcription 
Table 1

Viral protein specificity and isotypes of mAb's used in the study

\begin{tabular}{|c|c|c|c|c|c|c|}
\hline $\mathrm{mAb}$ & 7D9 & $8 \mathrm{D} 3$ & $1 \mathrm{E} 11$ & SGI & B4-2 & 159 \\
\hline Specificity & VP6 & VP6 & VP6 & VP6 & NSP4 & VP7 \\
\hline Isotype & $\lg A$ & $\lg A$ & $\lg G 2 a$ & $\lg G 2 a$ & $\lg G 1$ & $\lg G 1$ \\
\hline Classical neutralization titer & $<50$ & $<50$ & $<50$ & $<50$ & $<50$ & 500,000 \\
\hline
\end{tabular}

reaction products were resolved on a high-resolution denaturing $20 \%$ polyacrylamide gel and autoradiographed. For comparison purposes, equivalent quantities of native DLPs and mature TLPs were analyzed using the same procedures.

\section{Results}

Non-neutralizing anti-VP6 IgA mAb 7D9 effectively protects mice from rotavirus infection. Previously, in a "backpack" model in which mice were transplanted with hybridoma cells secreting selected mAb's, mAb 7D9 was shown to protect mice from virulent rotavirus challenge, while mice treated with other hybridoma cells failed to be protected (17). To rule out the possibility that the lack of protection was caused by variation in viability or Ig production in vivo by the transplanted hybridoma cells, we treated mice daily with $3 \mathrm{mg}$ of selected $\mathrm{mAb}$ injected intraperitoneally. The treated animals were challenged with wild-type murine rotavirus after 2 days of pretreatment as described. The protective efficacy of the antibodies expressed as the percent reduction of total fecal antigen shedding as compared with noninjected controls is presented in Figure 1. The non-neutralizing anti-VP6 IgA 7D9 completely protected mice from challenge, confirming our prior studies using the "backpack" model and a cell transfer approach (Figure 1). Even after a $1: 50$ dilution of ascites $(0.06 \mathrm{mg}$ daily dose), mice treated with 7D9 shed significantly less viral antigen than did controls (62\% reduction of shedding). Treatment with other non-neutralizing antibodies to VP6 or NSP4, including mAb 8D3, 1E11, SGI-255/60, and B4-2, did not significantly reduce fecal viral antigen shedding after challenge (Figure 1). Of note, VP7 serotype G3-specific neutralizing $\mathrm{mAb}$ 159 also failed to protect mice, even though the in vitro neutralizing titer against tissue culture-adapted EC virus was greater than 1:500,000 (Table 1). The protective efficacy of 7D9 was, however, affected by the dose size of challenge virus. When 7D9-treated mice received
$10^{5} \mathrm{ID}_{50}$ of $\mathrm{EC}$ virus rather than $10^{2} \mathrm{ID}_{50}$, no reduction of shedding was observed (data not shown). These findings confirm and extend our previous observations and indicate that the protective effect of 7D9 is not dependent on the transfer of living hybridoma cells to recipient mice but is dependent on the titer of the challenge dose administrated.

Lipofection-mediated intracellular neutralization. Because 7D9 is directed against the rotavirus intermediate layer protein VP6, which is only exposed on DLPs and is not the target of classical neutralization activity (Table 1 and ref. 17), we reasoned that the antibody may neutralize rotavirus only after the virus has entered the cell and has shed the outer capsid protein VP4 and VP7. Previous studies have shown that "noninfectious" DLPs were rendered infectious if introduced into the cytoplasm of permissive cells (30). Based on these results, we developed a lipofectionmediated intracellular neutralization assay to determine whether antibodies to VP6 had antiviral activity if introduced into the cytoplasm (see Methods). The intracellular neutralization titers of selected antibodies against a panel of rotaviruses including the challenge strain EC are provided in Table 2. Only mAb 7D9 effectively neutralized viruses at high titer in this lipofection-based assay. The neutralization titers of 7D9 were similar against the different virus strains tested, which represented a variety of serotype and subgroup specificities (Table 2). The nonprotective VP6 IgA antibody $8 \mathrm{D} 3$ had low intracellular neutralization titers, and this low-level neutralization was not consistently detectable. Antibodies 1E11 and B4-2 did not neutralize virus intracellularly, while mAb SGI-255/60 showed low neutralization activity against RRV only (a subgroup I virus). mAb 159, which effectively neutralizes infectious serotype $\mathrm{G} 3$ viruses in classical neutralization assay (Table 1), did not inhibit DLP viruses (Table 2) or TLP viruses (data not shown) in the lipofection assay. The reciprocal titers of the mAb's detect-

Table 2

Lipofection-mediated intracellular neutralization titers by selected mAb's

\begin{tabular}{|c|c|c|c|c|c|c|}
\hline \multirow[b]{2}{*}{ Virus neutralized (species) } & \multicolumn{6}{|c|}{ Titers (reciprocal) with indicated $\mathrm{mAb}$} \\
\hline & 7D9 & 8D3 & $1 \mathrm{E} 11$ & SGI & B4-2 & 159 \\
\hline $\mathrm{EC}$ (murine) & $5,120(2,560-10,240)$ & $40(<40$ to 160$)$ & $<40$ & $<40$ & $<40$ & $<40$ \\
\hline RRV (simian) & $5,120(2,560-10,240)$ & $50(<40$ to 160$)$ & $<40$ & $<40(<40$ to 40$)$ & $<40$ & $<40$ \\
\hline NCDV (bovine) & $2,560(640-10,240)$ & $40(<40$ to 160$)$ & $<40$ & $<40$ & $<40$ & $<40$ \\
\hline Wa (human) & $5,120(2,560$ to 10,240$)$ & $40(<40$ to 160$)$ & $<40$ & $<40$ & $<40$ & $<40$ \\
\hline
\end{tabular}

Mean intracellular neutralization titers and range (in parentheses) were calculated from two to four replicates. The differences between 7D9 neutralizing titers and the titers of all other groups were significant $(P<0.01)$. 


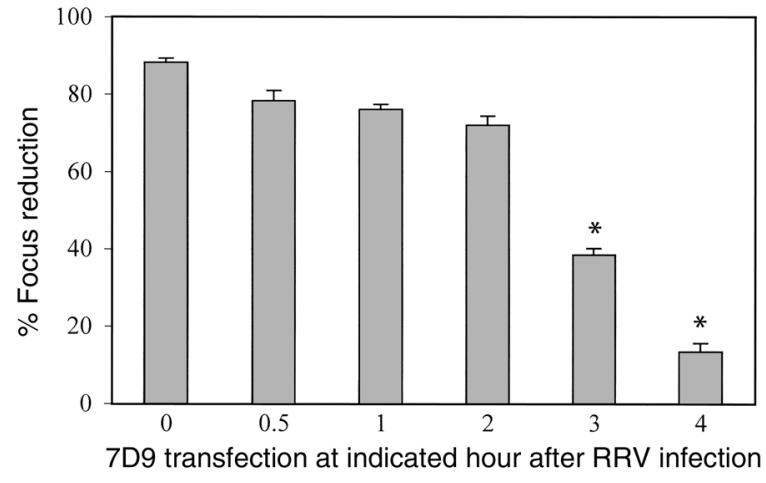

\section{Figure 2}

Efficiency of lipofection-mediated intracellular neutralization by 7D9 at different time points after infection. MA104 cells were infected with TLPs of RRV. At indicated time points after infection, 7D9 $\mathrm{mAb}$ was transfected into cells $(0.025 \mathrm{mg} / \mathrm{ml})$ using lipofectin. Viral antigen in the cells was detected 10 hours after initial RRV infection by immunostaining. Data are expressed as percentage of focus reduction as compared with control. *Focus reduction was below $50 \%$ and significantly different from that at time $0(P<0.05)$. Results were calculated from triplicate measurements and represent two separate experiments.

ed inside MA104 cells after transfection (data not shown) were between 2560 and 10,240 , suggesting that transfection efficiencies for different antibodies were similar and that the differences in intracellular neutralization activity were not due to differences in the amount of antibody transfected.

Kinetic of intracellular neutralization. To investigate at which stage of infection rotaviruses were being inhibited by IgA 7D9, we infected cells with RRV TLPs and, at selected times after infection, transfected purified 7D9 $(0.025 \mathrm{mg} / \mathrm{ml})$ into the infected monolayers (Figure 2). When 7D9 was transfected between 0 and 2 hours after infection, it significantly inhibited virus infection. However, by 3 hours after infection, reduction was less than $50 \%$ (below neutralizing titer cutoff) (Figure 2). The intracellular neutralizing activity became undetectable when 7D9 was transfected 4 hours after infection. Since the antibody was effective even when added to cells after infection, its effects are not likely to be due to extracellular aggregation.

In vitro inbibition of viral RNA transcription. The kinetic studies indicated that IgA 7D9 neutralizes rotavirus at an early stage of viral replication following viral infection. We hypothesized that IgA 7D9 may function, at least in part, by inhibiting viral mRNA transcription after the virus uncoats in the cytoplasm. To initially evaluate the inhibitory effects of selected rotavirusspecific antibodies on viral mRNA transcription, we incubated various antibodies with transcriptionally competent DLPs of RRV and EC and measured the incorporation of ${ }^{32} \mathrm{P}$ into viral mRNA transcripts (Figure 3 , $a$ and $b$ ). The level of transcription of RRV and EC in the presence of antibodies B4-2 and 159 was not significantly different from that of the control with no antibody added. The mRNA transcription of DLPs treated with antibodies 8D3 and 1E11 was significantly greater than that of the control. SGI slightly suppressed mRNA transcription of RRV only (62\%, $P<0.05)$. IgA 7D9, however, completely suppressed the in vitro rotavirus mRNA transcription of both RRV and EC (Figure 3, a and $\mathrm{b} ; P>0.01$ ). This finding is consistent with the hypothesis that IgA 7D9 mediates its antiviral effect by blocking early-phase transcription of the rotavirus replication cycle.

Three-dimensional structural analysis of $7 D 9$ DLP:IgA complexes. To directly visualize the interactions between 7D9 IgA and the VP6 capsid layer, we imaged DLP:IgA complexes in vitreous ice by electron cryomicroscopy (data not shown), computed the three-dimensional structure to a resolution of about $21 \AA$ from 137 independent particle images, and compared it with the structure of the native DLP (Figure 4). The VP6 capsid is made up of 260 trimers arranged on a $\mathrm{T}=13$ (levo) icosahedral lattice. This arrangement of capsomers defines 132 channels that penetrate the VP6 layer. In the outer regions of the VP6 capsid layer, the trimers are well separated from one another, while in the lower regions, near the VP2 layer, the trimers form a network of interactions with one another.

In the surface representation of the 7D9 DLP:IgA complex (Figure 4b), a total of 240 individual Fab domains (pink) were clearly visible on the viral surface. The Fab domains were attached at the tops of the VP6 trimers, with the epitope positioned along the edge. The Fab domains were oriented vertically on the viral

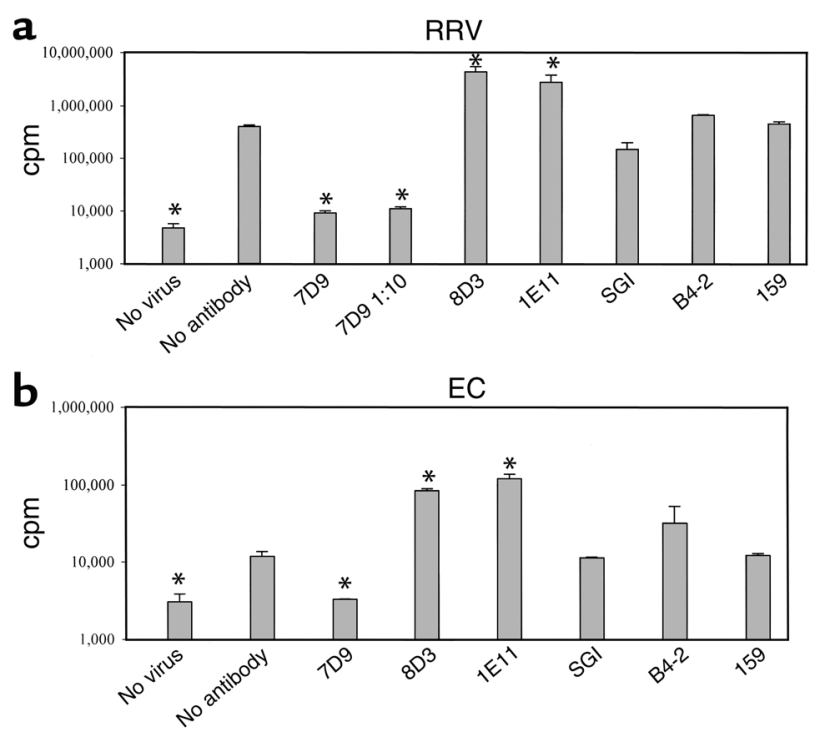

Figure 3

Effects of selected mAb's on in vitro rotavirus transcription. RRV (a) and EC $(\mathbf{b})$ were treated with EDTA and mixed with selected mAb's. Transcription assay was conducted using a Riboprobe system at $40^{\circ} \mathrm{C}$ for 1 hour as described. Transcripts were labeled with ${ }^{32} \mathrm{P} \mathrm{rCTP}$ and measured by liquid scintillation counter. Data are expressed as $\mathrm{cpm}$. As control, reactions with no virus or with virus but no antibody were also included. * Mean cpm in this group was significantly different from that of nonantibody control $(P<0.05)$. Results were calculated from four replicates and represent four separate experiments. 
surface and did not adversely affect the width of any of the channels penetrating the VP6 capsid layer.

The Fab domains had the expected bi-lobed morphology seen in the atomic model of $\operatorname{IgA}(40)$, and the molecular envelope was also similar to that observed for IgGs at a comparable resolution (22). Though bivalent IgA molecules were bound to the viral surface, only the Fab domains were visible in the three-dimensional structure. The most reasonable explanation for this is that both the Fc domains and the extended hinge regions, being highly flexible, were disordered and hence were averaged away during the reconstruction process.

Surrounding each icosahedral threefold axis was a cluster of three small spheres of mass density. The volume of each cluster was equivalent to that of an individual Fab domain, suggesting that one Fab domain is also bound to the VP6 trimer located at each of the 20 icosahedral threefold axes, though the morphology cannot be visualized in the three-dimensional structure. A Fab domain interacting with a trimer at the icosahedral threefold axis may adopt any one of three different orientations, depending upon which underlying VP6 monomer is specifically bound. The disrupted morphology of these Fab domains in the threedimensional structure is likely a consequence of the fact that only one of three symmetrically equivalent positions is occupied around each icosahedral threefold axis. Taken together, these results indicate that a total of 260 IgA antigen-binding domains, or one per VP6 trimer, are bound to the VP6 surface at saturation.

Comparison of the VP6 capsid region in the 7D9 DLP:IgA complex and the native $D L P$. The attachment of various ligands, such as the VP7 capsid protein or anti-VP6 Fab's, to the VP6 surface has been reported to introduce architectural changes in the body of the VP6 capsid layer (22). In these studies, mass translocations were observed within the network of trimer-trimer interactions present near the VP6-VP2 interface region in the vicinity of the mRNA release channels at the icosahedral fivefold axes. To investigate whether the attachment of 7D9 IgA causes similar structural changes in VP6, we compared the distribution of mass in the native DLP and the 7D9 DLP:IgA complex at various radii within the VP6 capsid region.

In the vicinity of the mRNA release channels (Figure 5 ), structural differences were evident at nearly all radii examined, and the types of domain movements were very similar to those observed in previous structural studies of DLP-ligand complexes (22). As in previous studies, the most striking mass translocations were observed within the network of trimer-trimer interactions extending between the radii $\sim 275 \AA \AA$ to $\sim 310 \AA$. In this region, individual molecules of VP6 appeared to be shifted with respect to each other, with some displacements exceeding $10 \AA$ (Figure 5a, section III). As a result of these movements, some of the intermolecular interactions within trimers appeared to be weakened, and some of the intermolecular interactions between trimers appeared to grow stronger. Furthermore, the movement of the VP6 molecules closest to the icosahedral fivefold axes caused the diameter of the mRNA release channel to narrow by about $10 \AA$ in the middle of this region. Mass translocations were not observed at the present resolution in the VP6-VP2 interface ( $260 \AA$ A; Figure $5 \mathrm{a}$, section $\mathrm{V}$ ) or the VP2 capsid layer (data not shown).

Interestingly, the sorts of mass translocations observed within the VP6 trimers surrounding the mRNA release channels did not occur in other regions of the capsid (data not shown). The distribution of mass within the VP6 trimers surrounding the icosahedral twofold axes was virtually identical in both the native DLP and the 7D9 DLP:IgA complex structures. Likewise, few structural changes were observed within the VP6 trimers located at other positions examined. The preponderance of structural changes in the regions of VP6 immediately adjacent to the mRNA release channels suggests that the trimers near the icosahedral vertices are more flexible than trimers at other locations and are more susceptible to alteration upon ligand attachment.

Analysis of transcription products in 7D9 DLP:IgA complex. In previous structural and biochemical studies of DLPligand complexes, the appearance of architectural rearrangements in the VP6 capsid layer directly correlated with an inhibition of genome transcription at the stage of transcript elongation $(21,22)$. Our initial studies indicated that 7D9 efficiently inhibited transcription of RRV and EC while other anti-VP6 mAb's did not. To determine whether the attachment of 7D9 IgA to the DLP surface likewise blocks elongation but not

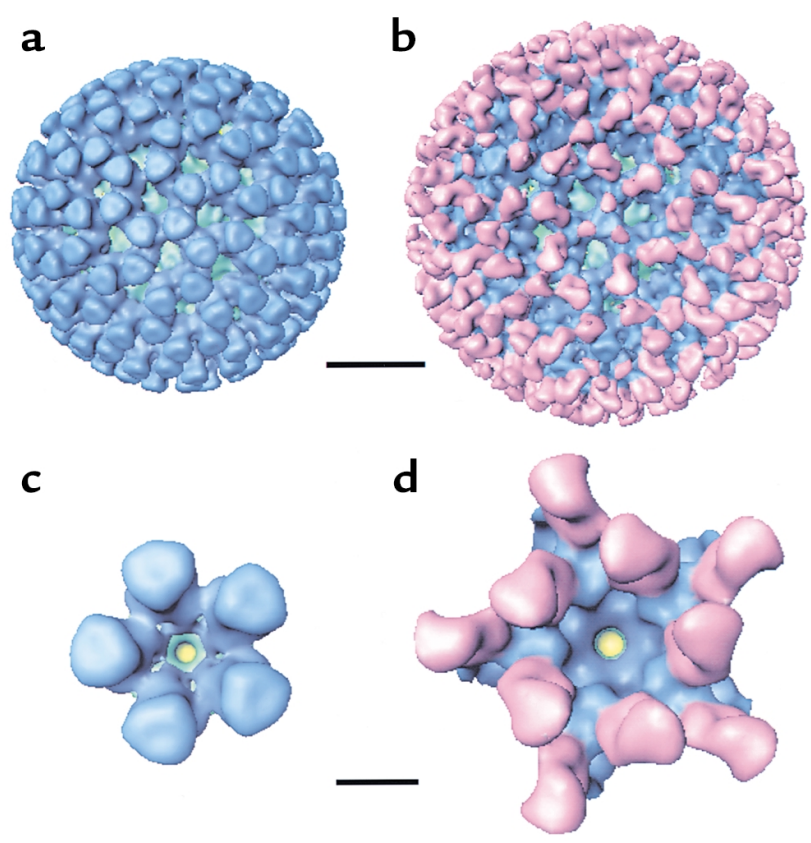

Figure 4

( $\mathbf{a}$ and $\mathbf{b}$ ) Surface representations of the three-dimensional structure of native DLP (a) and 7D9 DLP:IgA complex (b), viewed along the icosahedral threefold axis. The VP2 capsid layer is shown in green, VP6 in blue, and IgA in pink. Scale bar, $200 \AA$. (c and d) Surface representations of the $m R N A$ release channel computationally isolated from the native DLP (c) and the 7D9 DLP:IgA complex (d). Scale bar, $50 \AA$. 

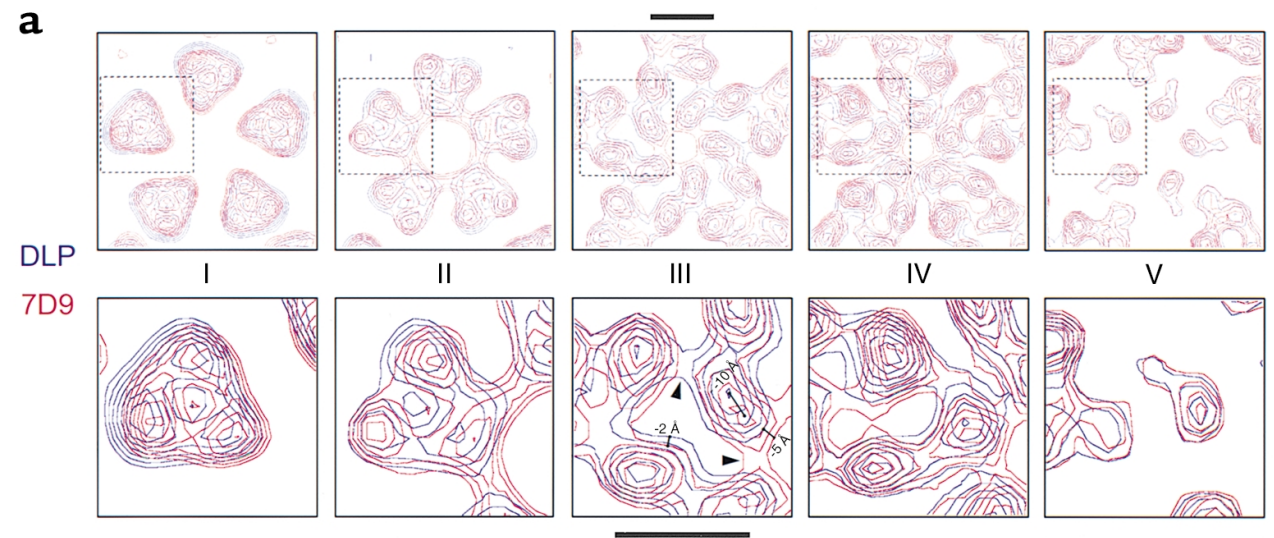

b
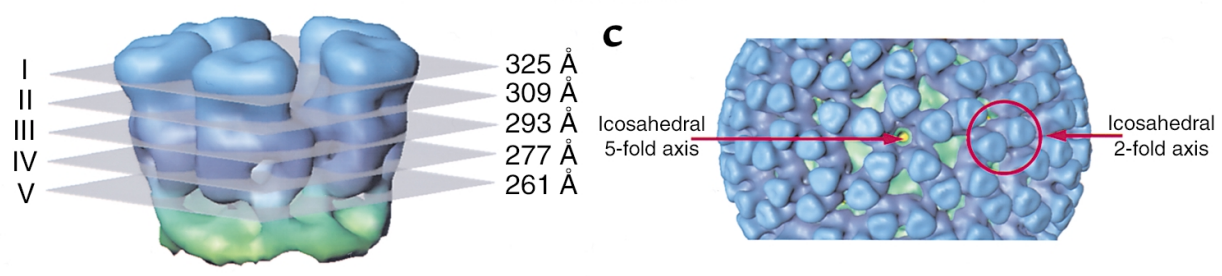

\section{Figure 5}

(a) Contoured cross sections of mass density from the three-dimensional structures of native DLP (blue) and 7D9 DLP:IgA complex (red), viewed perpendicular to the icosahedral fivefold axis. Each contour represents a mass density difference of about $0.37 \sigma$. The cross sections, labeled I-V, were extracted from radii between $261 \AA$ and $325 \AA$ (b). The upper panel of cross sections depicts the distribution of mass surrounding the mRNA release channel, with the icosahedral fivefold axis passing through the center. The lower panel of cross sections provides a close-up view of the mass distribution in one of the five trimers (indicated in the corresponding upper panel by a dashed box). The arrowheads in cross section III, lower panel, denote representative intermolecular interactions that are different in the two structures. Measurements of three specific mass translocations are also given. Scale bars, $50 \AA$. (b) Graphical representation of the radii from which cross sections were extracted in a. The cross sections are shown as semitransparent gray planes. The five VP6 trimers surrounding the mRNA release channel in the native DLP are colored in blue, and the underlying VP2 capsid layer is shown in green. (c) A portion of the surface representation of the native DLP, viewed along the icosahedral fivefold axis. The position of the fivefold axis along which the sections shown in a were taken is marked. The pair of VP6 trimers surrounding the twofold axis is also indicated.

initiation of transcription, we next compared the length distribution of transcription products in native DLPs and 7D9 DLP:IgA complexes following a period of incubation in a standard in vitro transcription reaction buffer (Figure 6). While native DLPs, as expected, were able to produce full-length mRNA transcripts (lane 1), 7D9 DLP:IgA complexes synthesized predominantly short oligonucleotide transcripts having an apparent length of about six nucleotides and only a small quantity of full-length mRNA (lane 2). The transcriptional behavior of the 7D9 DLP:IgA complex is similar to that of the mature TLP, which is not able to elongate mRNA transcripts beyond about 6 bases in length (lane 3). Given the similarities between the mature TLP and the 7D9 DLP:IgA complex in regard to the architecture of the VP6 capsid region, these results suggest that 7D9 IgA causes an inhibition of genome transcription by the same mechanism as does VP7 in the mature particle.

\section{Discussion}

Rotavirus surface proteins VP4 and VP7 have long been identified as targets of protective neutralizing antibody both in vitro and in vivo (4-7). Recently, studies have shown that rotavirus intermediate capsid protein VP6 can also stimulate a protective immune response (12-16). Unlike the two viral surface proteins VP4 and VP7, which exhibit different serotypes, VP6 of all group A rotaviruses shares a high degree of antigenic cross-reactivity and could potentially be responsible for a component of the heterotypic protection observed following rotavirus infection. It should be noted, however, that both VP4 and VP7 contain crossreactive as well as homotypic neutralization epitopes. Hence the heterotypic component of immunity-generated posthomotypic infection is certainly produced, at least in part, by anti-VP4 and -VP7 responses.

Previously, we described several anti-VP6 IgA mAb's that protected mice from virulent rotavirus challenge and that cleared virus shedding in chronically infected SCID mice (17). The protection did not appear to be dependent on classical neutralization activity, since the anti-VP6 antibodies did not neutralize virus in vitro and did not protect if fed orally to mice. In fact, the intermediate capsid protein VP6 is virtually inaccessible to all but small molecules in the mature, triple-layered, infectious particle. We hypothesized that the anti-VP6 IgA antibodies might interact with rotavirus DLPs inside intestinal epithelial cells and neutralize the virus intracellularly.

Intestinal secretory $\operatorname{IgA}(\operatorname{IgA})$ is the major adaptive immune component in defense of the mucosal surface. 


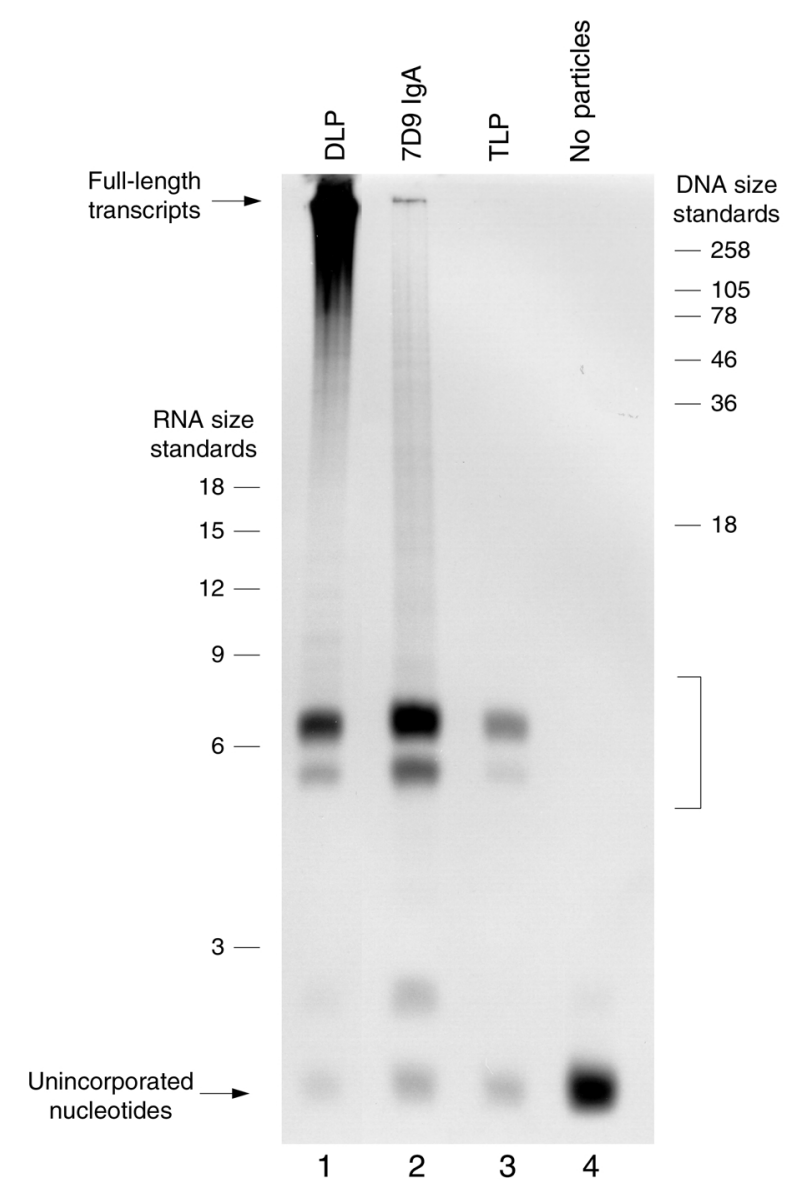

\section{Figure 6}

Denaturing PAGE analysis of transcription products in the native DLP (lane 1), 7D9 DLP:IgA complex (lane 2), mature TLP (lane 3), and control reaction containing no particles (lane 4). The positions of full-length mRNA and unincorporated nucleotides are noted with arrows. The position of short oligonucleotide transcription products in lanes 1-3 is marked with a bracket. The band corresponding to unincorporated nucleotides at the bottom of the autoradiograph is not of the same intensity in each reaction because of the differing efficiency with which gel filtration columns removed the nucleotide precursors after transcription.

In addition to neutralizing pathogens by inhibiting their attachment and entry into cells, several authors have reported that sIgA can also neutralize various viruses such as HIV, influenza, and Sendai virus intracellularly during transcytosis (41-43). We carried out a series of functional, biochemical, and structural studies to investigate potential mechanisms of intracellular neutralization by anti-VP6 IgA mAb's. Using a lipofectin-mediated intracellular neutralization assay, we demonstrated that the IgA mAb 7D9 inhibited viral replication after being transfected into MA104 cells with virus DLPs, which are noninfectious without transfection. Other rotavirus-specific mAb's were ineffective or only minimally effective in this assay. Rotaviruses with different serotypes isolated from different animal or human species were all neutralized by 7D9 at similar titers, suggesting that the antibody had broad het- erotypic activity. Moreover, the neutralization efficiency was greatly diminished if 7D9 was transfected more than 4 hours after virus infection, indicating that the antibody is active at an early stage of viral replication.

Several different potential mechanisms of intracellular neutralization have been reported previously. Bomsel et al. (41) described the inhibition of HIV transcytosis across the tight epithelial barrier by IgA or IgM antibodies directed against the viral envelope. Non-hemagglutination inhibition influenza hemagglutinin-specific antibodies were shown to prevent influenza virus fusion with the intracellular vacuolar membrane (44). Reovirus uncoating can be blocked by antibodies against outer capsid protein (45). Antibody 7D9, which is specific for rotavirus double-layered particles, could potentially block steps, such as transcription, that occur after uncoating.

In vitro biochemical characterization of IgA-bound DLPs provides additional evidence that 7D9 may be inhibiting rotavirus replication by blocking the genome transcription phase of the virus life cycle. In contrast to other IgA and IgG mAb's examined, 7D9 completely inhibited the production of mature mRNA transcripts in two rotavirus strains. It is not clear why some antiVP6 antibodies actually appear to enhance transcription, and indeed this phenomenon warrants further structural analysis. Furthermore, analysis of the transcription products synthesized within 7D9 DLP:IgA complexes indicated that transcript elongation, rather than initiation, was specifically inhibited by the attachment of IgA on the viral surface. In this respect, the transcriptional behavior of the 7D9 DLP:IgA complex is nearly identical to that of the mature, infectious virion.

Following IgA binding, the conformation of the VP6 capsid layer exhibits a number of changes when compared with the native DLP as examined by cryoelectron microscopy and image reconstruction. These architectural changes, some of which involve mass movements exceeding $10 \AA$, appear to be concentrated within the trimers surrounding the icosahedral vertices. These conformational changes affect intermolecular interactions both within and between trimers. As a consequence of these mass translocations, the diameter of the mRNA release channel is narrowed by about 10 Å near the VP6VP2 interface. Similar differences were previously observed in another transcriptionally incompetent antibody-bound DLP structure as well as in the mature TLP (22). Indeed, such architectural changes appear to correlate with the inhibition of transcription elongation, suggesting that 7D9 IgA likely inhibits transcription by the same mechanism as does VP7 in the mature TLP.

The precise mechanism of transcription inhibition is not readily apparent from these architectural changes. While transcription inhibition could potentially result directly from ligand-induced changes in the architecture of the capsid, not all transcriptionally incompetent antibody-DLP complexes studied using structural techniques show evidence of conformational changes in the VP6 capsid layer upon anti- 
body binding, suggesting that perhaps the precise site of attachment and a potential loss of flexibility represent important factors as well (46). Conceivably, multiple mechanisms may exist by which an antibody attached to the VP6 surface could prevent transcription of the genome. Whether or not ligand attachment introduces noticeable conformational changes, the conformation of the VP6 capsid layer during transcription is evidently critical for the process to occur efficiently, and even subtle changes in either the capsid architecture or the flexibility of the trimers may be enough to prevent transcript elongation from taking place, leading to interruption of the virus life cycle.

It is not known how many antibody molecules are minimally required to provide effective intracellular neutralization of rotavirus. At full saturation, 240 7D9 IgA antigen-binding domains can interact with the surface of the DLP. However, because the mechanism of transcription inhibition likely results from the introduction of subtle conformational changes in the capsid, it is conceivable that far fewer than 240 antigenbinding domains may be sufficient to cause a block in transcript elongation, particularly if those antibodies are bound in the vicinity of the icosahedral vertices where mRNA transcripts exit the particle. It is also conceivable that as few as several antibodies bound within the vicinity of one or two of the vertices might be able to prevent elongation of specific transcripts, leading to a deficit of one or more viral proteins and consequently a block in replication equivalent to that of a mutant particle lacking one or more genome segments. Were such a mechanism to operate, however, it could likely be overcome by the presence of multiple infectious particles in the same cell, as would be the case with high doses of challenge virus. Indeed, our results indicate that the protective efficacy of 7D9 IgA is dependent upon the titer of the challenge virus.

Our functional and structural studies provide a mechanism to explain how IgA molecules directed against specific epitopes on the intermediate capsid protein VP6 could function in vivo to provide protection against rotavirus infection by disrupting genome transcription following cell entry. Although our studies have not specifically examined whether 7D9 antibodies are present in the cytoplasm of infected enterocytes in vivo, our findings do strongly suggest that 7D9 antibodies exert their protective effect intracellularly by inhibiting genome transcription. In the case of 7D9 IgA activity in vitro, disruption of viral replication is most effective when IgA is present in the cytoplasm of the infected cell during the first 2 hours following cell entry, a time window that coincides with genome transcription. Inhibition of transcription occurs at the stage of transcript elongation and is accompanied by ligand-induced conformational changes in the viral capsid, just as is observed in the mature TLP where VP7 is bound, suggesting a similar mechanism of inhibition. Taken together, these results suggest that antibody-mediated disruption of viral replication downstream of cell entry may be an important immune strategy in combatting rotavirus infection. Further studies are being conducted to determine whether 7D9 can be identified in the cytoplasm of infected enterocytes. In addition, future studies are needed to investigate the mechanism by which 7D9 might enter the infected cell and the manner in which antibody present in a transcytotic vesicle might come in contact with input virus.

\section{Acknowledgments}

This work was supported by NIH grants AI-36040 and AI-21632 (to B.V.V. Prasad), and DK-38707 and DK56339 (to H.B. Greenberg), and a grant from the Veterans Administration to H.B. Greenberg.

1. Bresee, J.S., Glass, R.I., Ivanoff, B., and Gentsch, J.R. 1999. Current status and future priorities for rotavirus vaccine development, evaluation and implementation in developing countries. Vaccine. 17:2207-2222.

2. Lawton, J.A., Estes, M.K., and Prasad, B.V. 2000. Mechanism of genome transcription in segmented dsRNA viruses. Adv. Virus Res. 55:185-229.

3. Estes, M.K. 2001. Rotaviruses and their replication. In Fields virilogy. 4th edition. D.M. Knippe et al., editors. Lippincott Williams \& Wilkins. Philadelphia, Pennsylvania, USA. 1747-1786.

4. Greenberg, H.B., et al. 1983. Production and preliminary characterization of monoclonal antibodies directed at two surface proteins of rhesus rotavirus. J. Virol. 47:267-275.

5. Matsui, S.M., et al. 1989. Passive protection against rotavirus-induced diarrhea by monoclonal antibodies to the heterotypic neutralization domain of VP7 and the VP8 fragment of VP4. J. Clin. Microbiol. 27:780-782.

6. Matsui, S.M., Mackow, E.R., and Greenberg, H.B. 1989. Molecular determinant of rotavirus neutralization and protection. Adv. Virus Res. 36:181-214.

7. Offit, P.A., and Blavat, G. 1986. Identification of the two rotavirus genes determining neutralization specificities. J. Virol. 57:376-378.

8. Vesikari, T. 1993. Clinical trials of live oral rotavirus vaccines: the Finnish experience. Vaccine. 11:255-261.

9. Ward, R.L., et al. 1992. Evidence that protection against rotavirus diarrhea after natural infection is not dependent on serotype-specific neutralizing antibody. J. Infect. Dis. 166:1251-1257.

10. Ward, R.L., McNeal, M.M., and Sheridan, J.F. 1992. Evidence that active protection following oral immunization of mice with live rotavirus is not dependent on neutralizing antibody. Virology. 188:57-66.

11. Feng, N., et al. 1997. Heterotypic protection following oral immunization with live heterologous rotaviruses in a mouse model. J. Infect. Dis. 175:330-341.

12. Chen, S.C., et al. 1997. Protective immunity induced by rotavirus DNA vaccines. Vaccine. 15:899-902.

13. Ciarlet, M., et al. 1998. Subunit rotavirus vaccine administered parenterally to rabbits induces active protective immunity. J. Virol. 72:9233-9246.

14. Herrmann, J.E., et al. 1996. Protection against rotavirus infections by DNA vaccination. J. Infect. Dis. 1:S93-S97.

15. O'Neal, C.M., Crawford, S.E., Estes, M.K., and Conner, M.E. 1997. Rotavirus virus-like particles administered mucosally induce protective immunity. J. Virol. 71:8707-8717.

16. Siadat-Pajouh, M., and Cai, L. 2001. Protective efficacy of rotavirus 2/6virus-like particles combined with CT-E29H, a detoxified cholera toxin adjuvant. Viral Immunol. 14:31-47.

17. Burns, J.W., Siadat, P.M., Krishnaney, A.A., and Greenberg, H.B. 1996. Protective effect of rotavirus VP6-specific IgA monoclonal antibodies that lack neutralizing activity. Science. 272:104-107.

18. Lawton, J.A., Estes, M.K., and Prasad, B.V. 1997. Three-dimensional visualization of mRNA release from actively transcribing rotavirus particles. Nat. Struct. Biol. 4:118-121.

19. Cohen, J., Laporte, J., Charpilienne, A., and Scherrer, R. 1979. Activation of rotavirus RNA polymerase by calcium chelation. Arch. Virol. 60:177-186.

20. Sandino, A.M., Jashes, M., Faundez, G., and Spencer, E. 1986. Role of the inner protein capsid on in vitro human rotavirus transcription. J. Virol. 60:797-802.

21. Lawton, J.A., Estes, M.K., and Prasad, B.V. 2001. Identification and characterization of a transcription pause site in rotavirus. J. Virol. 75:1632-1642.

22. Lawton, J.A., Estes, M.K., and Prasad, B.V. 1999. Comparative structural analysis of transcriptionally competent and incompetent rotavirus-anti- 
body complexes. Proc. Natl. Acad. Sci. USA. 96:5428-5433.

23. Corthesy, B., and Spertini, F. 1999. Secretory immunoglobulin A: from mucosal protection to vaccine development. Biol. Chem. 380:1251-1262.

24. Falconer, M.M., Gilbert, J.M., Roper, A.M., Greenberg, H.B., and Gavora, J.S. 1995. Rotavirus-induced fusion from without in tissue culture cells. J. Virol. 69:5582-5591.

25. Ward, R.L., and Ashley, C.S. 1980. Comparative study on the mechanisms of rotavirus inactivation by sodium dodecyl sulfate and ethylenediaminetetraacetate. Appl. Environ. Microbiol. 39:1148-1153.

26. Feng, N., Burns, J.W., Bracy, L., and Greenberg, H.B. 1994. Comparison of mucosal and systemic humoral immune responses and subsequen protection in mice orally inoculated with a homologous or a heterologous rotavirus. J. Virol. 68:7766-7773.

27. Petrie, B.L., Greenberg, H.B., Graham, D.Y., and Estes, M.K. 1984. Ultrastructural localization of rotavirus antigens using colloidal gold. Virus Res. 1:133-152.

28. Shaw, R.D., Vo, P.T., Offit, P.A., Coulson, B.S., and Greenberg, H.B. 1986 Antigenic mapping of the surface proteins of rhesus rotavirus. Virology. 155:434-451.

29. Ishida, S., Feng, N., Tang, B., Gilbert, J.M., and Greenberg, H.B. 1996 Quantification of systemic and local immune responses to individual rotavirus proteins during rotavirus infection in mice. J. Clin. Microbiol. 34:1694-1700.

30. Bass, D.M., et al. 1992. Liposome-mediated transfection of intact viral particles reveals that plasma membrane penetration determines permissivity of tissue culture cells to rotavirus. J. Clin. Invest. 90:2313-2320.

31. Cohen, J., and Dobos, P. 1979. Cell free transcription and translation of rotavirus RNA. Biochem. Biophys. Res. Commun. 88:791-796.

32. Dubochet, J., et al. 1988. Cryo-electron microscopy of vitrified specimens. Q. Rev. Biophys. 21:129-228.

33. Lawton, J.A., and Prasad, B.V. 1996. Automated software package for icosahedral virus reconstruction. J. Struct. Biol. 116:209-215.

34. Crowther, R.A., DeRosier, D.J., and Klug, A. 1970. The reconstruction of a three-dimensional structure from projections and its applications to electron microscopy. Proc. R. Soc. Lond. A317:319-340.
35. Crowther, R.A. 1971. Procedures for three-dimensional reconstruction of spherical viruses by Fourier synthesis from electron micrographs. Philos. Trans. R. Soc. Lond. B Biol. Sci. 261:221-230.

36. Fuller, S.D. 1987. The $\mathrm{T}=4$ envelope of Sindbis virus is organized by interactions with a complementary $\mathrm{T}=3$ capsid. Cell. 48:923-934.

37. Olson, N.H., and Baker, T.S. 1989. Magnification calibration and the determination of spherical virus diameters using cryo-microscopy. Ultramicroscopy. 30:281-297.

38. Zhou, H., and van Oosterom, A. 1994. Application of the boundary element method to the solution of anisotropic electromagnetic problems. Med. Biol. Eng. Comput. 32:399-405.

39. van Heel, M. 1987. Similarity measures between images. Untramicroscopy. 21:95-99.

40. Boehm, M.K., Woof, J.M., Kerr, M.A., and Perkins, S.J. 1999. The Fab and Fc fragments of IgA1 exhibit a different arrangement from that in IgG: a study by X-ray and neutron solution scattering and homology modeling. J. Mol. Biol. 286:1421-1447.

41. Bomsel, M., et al. 1998. Intracellular neutralization of HIV transcytosis across tight epithelial barriers by anti-HIV envelope protein $\operatorname{IIgA}$ or IgM. Immunity. 9:277-287.

42. Mazanec, M.B., Kaetzel, C.S., Lamm, M.E., Fletcher, D., and Nedrud, J.G. 1992. Intracellular neutralization of virus by immunoglobulin A antibodies. Proc. Natl. Acad. Sci. USA. 89:6901-6905.

43. Mazanec, M.B., Coudret, C.L., and Fletcher, D.R. 1995. Intracellular neutralization of influenza virus by immunoglobulin A anti-hemagglutinin monoclonal antibodies. J. Virol. 69:1339-1343.

44. Imai, M., Sugimoto, K., Okazaki, K., and Kida, H. 1998. Fusion of influenza virus with the endosomal membrane is inhibited by monoclonal antibodies to defined epitopes on the hemagglutinin. Virus Res. 53:129-139.

45. Virgin, H.W., 4th, Mann, M.A., and Tyler, K.L. 1994. Protective antibodies inhibit reovirus internalization and uncoating by intracellular proteases. J. Virol. 68:6719-6729.

46. Thouvenin, E., et al. 2001. Antibody inhibition of the transcriptase activity of the rotavirus DLP: a structural view. J. Mol. Biol. 307:161-172. 